\title{
Prevalence, Characteristics and Antibiogram Profiles of Escherichia coli Isolated from Apparently Healthy Chickens in Mymensingh, Bangladesh
}

\author{
ATM Jakaria, Md. Ariful Islam*, Mst. Minara Khatun \\ Department of Microbiology and Hygiene, Faculty of Veterinary Science, Bangladesh Agricultural University, \\ Mymensingh-2202, Bangladesh. \\ *Corresponding author's e-mail: arifmicro2003@yahoo.com
}

[Received: 03 May 2012, Revised: 15 June 2012, Accepted: 20 June 2012]

\begin{abstract}
A B S T R A C T
Escherichia coli known to cause food-borne illnesses worldwide that are closely associated with the consumption of contaminated poultry and egg products. This study was undertaken for cultural, biochemical and antibiotic sensitivity analyses of $E$. coli recovered from apparently healthy chickens. Cloacal samples $(\mathrm{n}=350)$ were aseptically collected from layers $(\mathrm{n}=150)$, broilers $(\mathrm{n}=150)$ and indigenous chickens $(\mathrm{n}=50)$. The samples were enriched in nutrient broth and streaked onto eosin methylene blue (EMB) agar, MacConkey (MC) agar, blood agar, salmonella-shigella (SS) agar and brilliant green agar (BGA) for cultural characterization of the E. coli isolates. Culture-positive samples yielded characteristic colonies of E. coli with metallic sheen on EMB agar, bright pink or red colonies on MC agar, hemolysis on blood agar, slight pink smooth colonies on SS agar and green color colonies on BGA media. The E. coli isolates produced acid and gas by fermenting sugars (dextrose, sucrose, lactose, maltose and mannitol) and gave positive reaction to indole, methyl red (MR) and catalase tests, but were negative to Voges-Proskauer (VP) test. The prevalence of $E$. coli in layers, broilers and indigenous chickens were 78.67, 82 and 70\%, respectively. The antibiotic sensitivity pattern demonstrated that $E$. coli isolates were mostly sensitive to ciprofloxacin, gentamicin and cephalexin, and resistant to streptomycin, tetracycline, amoxicillin and nalidixic acid. Data of this study suggested that intestine of chicken could be a major reservoir of antibiotic resistant $E$. coli.
\end{abstract}

Keywords: Escherichia coli, Prevalence, Characteristics, Chickens, Antibiogram profiles

(C) 2012 Microbes and Health. All rights reserved

\section{Introduction}

Escherichia coli are Gram negative bacteria under the family Enterobacteriaceae. E. coli are widely distributed in nature, being present in soil and surface water and in animal and human feces. The most important reservoir of E. coli is the intestinal tract of animals and poultry (Bélanger et al., 2011). In addition, it is present on the bird's skin and feathers. These bacteria cause colibacillosis in chickens which is characterized by air sacculitis, cellulitis, omphalitis, peritonitis, salpingitis, synovitis and coligranuloma. This disease has an important economic impact on poultry production worldwide. The majority of economic losses results from mortality and loss of productivity of the affected birds (Otaki et al., 1995).

E. coli strains always belong to both pathogenic and nonpathogenic types (Pupo et al., 1997). In the caecal flora of healthy chickens, $10-15 \%$ of the E. coli strains may belong to an O-serotype. As soon as the first hour after hatching, the birds start building up their $E$. coli flora. The

To cite this article: Jakaria ATM, MA Islam and MM Khatun, 2012. Prevalence, characteristics and antibiogram profiles of escherichia coli isolated from apparently healthy chickens in Mymensingh, Bangladesh. Microbes Health, 1(1): 27-29. bacteria drastically increase their numbers in the gut. Birds may be infected with $E$. coli from contaminated environment, direct contact with sick birds, feces, water and feed (Dho-Moulin et al., 1999). Rodents may act as a carrier of avian pathogenic E. coli (APEC) and hence a source of contamination for the birds (Barnes et al., 1997)

Most of the strains of E. coli are harmless but some strains can cause food poisoning. E. coli food poisoning is usually caused by: enteropathogenic E. coli (EPEC) enterotoxigenic E. coli (ETEC); enterinvasive (EIEC) and enterohemorrhagic E. coli (EHEC) (Mead et al., 1999). E. coli enters the body through the consumption of food containing bacteria. Eating of inadequately cooked meat is the most common way that causes $E$. coli food poisoning. Raw poultry meat may contain $E$. coli that can cause food poisoning (Gormley et al., 2011). When a chicken is eviscerated, the healthy parts can be easily contaminated. Food animal such as cattle, pig and chickens appear to be reservoir of this organism (Geser et al., 2012). Multidrug resistance E. coli are produced due to use of antibiotics in animal's feeds as well as incomplete course of treatment against $E$. coli infection of humans and animals (Marshall et al., 1990). The present study was undertaken to determine the prevalence and characteristics of $E$. coli in apparently healthy chickens at farms, live bird markets and villages of Mymensingh area in Bangladesh. 


\section{Materials and Methods}

\section{Sample collection}

Swab samples were aseptically collected from apparently healthy layers of Bangladesh Agricultural University (BAU) Poultry Farm $(\mathrm{n}=100)$ and Boira Poultry Farm $(\mathrm{n}=50)$, broilers at Kamal Ranjit $(\mathrm{KR})$ market $(\mathrm{n}=50)$, BAU Sesh-more market $(n=50)$, and Kewatkhali market $(\mathrm{n}=50)$ and indigenous chickens at Boira village $(\mathrm{n}=50)$, Mymensingh. Samples were obtained from 16-18 weeks old laying hens, 10-12 weeks old broilers and 12-16 weeks old indigenous chickens.

\section{Enrichment}

Immediately after collection, samples were separately enriched in Nutrient Broth (NB) by incubating at $37^{\circ} \mathrm{C}$ for overnight

\section{Isolation of $E$. coli}

After enrichment in NB, a small amount of inoculum from $\mathrm{NB}$ was streaked duplicate onto eosin methylene blue (EMB) agar media and incubated at $37^{\circ} \mathrm{C}$ for overnight (Cheesbrough, 1985).

\section{Characterization of $\boldsymbol{E}$. coli}

Characterization of the $E$. coli was performed on the basis of cultural characteristics, Gram staining, motility test and antibiogram profiles. Colonial morphology of E. coli such as- size, margin, elevation and color were recorded on EMB agar, nutrient agar (NA), brilliant green agar (BGA), blood agar, salmonella-shigella (SS) agar, triple sugar iron (TSI) agar and MacConkey (MC) agar media to study cultural characteristics (Cheesebourgh,1985). Biochemical characterization of $E$. coli was performed by sugar fermentation reactions. Also, methyl red-Voges Proskauer (MRVP), catalase and indole tests were done according to the method described by Cowan (1985).

\section{Antibiogram study}

Ten $E$. coli isolates were randomly selected for antibiotic sensitivity assay. A previously described modified disc diffusion or Kirby-Bauer method (Bauer et al., 1966) was used to determine the susceptibility of $E$. coli isolates against antibiotic agents. In brief, the procedure involved measuring the diameter of the zone of inhibition that results from diffusion of the antimicrobial agents into the medium surrounding the disc. The reactions of test organisms to each antibiotic were classified as sensitive, intermediate and resistant according to the diameter of zone of inhibition recommended by NCCLS (2003). Ten commercially available antimicrobial discs (Oxoid Ltd, Baringstoke, Hampshire, England) were used in this study, which are mentioned with corresponding standard disc concentration in Table 1.

Table 1. Antibiotic concentration of discs used in antibiotic sensitivity assay for $\hat{E}$. coli

\begin{tabular}{lc}
\hline Name of antibiotic & Concentration per disc $(\boldsymbol{\mu g})$ \\
\hline Chloramphenicol & 30 \\
Ciprofloxacin & 5 \\
Gentamicin & 10 \\
Cephalexin & 30 \\
Kanamycin & 30 \\
Cephradine & 30 \\
Amoxicillin & 10 \\
Streptomycin & 10 \\
Tetracycline & 30 \\
Nalidixic acid & 30 \\
\hline
\end{tabular}

\section{Results}

Prevalence of $E$. coli

The overall prevalence was $78.86 \%$. The prevalence of $E$. coli in different chicken lines from all sources in this study are presented in Table 2 .

\section{Cultural, staining and motility characteristics}

E. coli on EMB agar produced greenish black colonies with metallic sheen. On MC agar, the colonies were bright pink in color, transparent, smooth and raised. Hemolysis was produced in blood agar media. Slight pink smooth colonies were seen on SS agar and green color colonies were found on BGA media. The organisms appeared Gram negative, small rod shaped and arranged in single, pair or short chain. All the isolates were found to be motile.

\section{Biochemical tests}

The five basic sugars viz. dextrose, sucrose, lactose, maltose and mannitol were fermented by all the isolates with the production of acid and gas. Acid production was indicated by the color change from reddish to yellow and the gas production was seen by the appearance of gas bubbles in the inverted Durham's tubes. All E. coli isolates were catalase, indole and MR positive but VP negative.

\section{Antibiogram profiles}

Antibiotic sensitivity tests of chicken $E$. coli isolates revealed as sensitive, intermediate and resistant profiles which are shown in Table 3.

Table 2. Prevalence of $E$. coli in layers, broilers and indigenous chickens

\begin{tabular}{llll}
\hline $\begin{array}{l}\text { Chicken } \\
\text { lines (n) }\end{array}$ & $\begin{array}{l}\text { E. coli positive } \\
\text { samples (n) }\end{array}$ & $\begin{array}{l}\text { Prevalence } \\
(\boldsymbol{\%})\end{array}$ & $\begin{array}{l}\text { Overall } \\
\text { prevalence (\%) }\end{array}$ \\
\hline Layers (150) & 118 & 78.67 & \\
Broilers (150) & 123 & 82.00 & 78.86 \\
Indigenous (50) & 35 & 70.00 & \\
\hline
\end{tabular}

Table 3. Antibiotic sensitivity pattern of the E. coli isolates from healthy chickens

\begin{tabular}{lccc}
\hline \multirow{2}{*}{$\begin{array}{l}\text { Name of } \\
\text { antibiotics }\end{array}$} & \multicolumn{3}{c}{ Antibiotic sensitivity profiles } \\
\cline { 2 - 4 } Ciprofloxacin & Sensitive & Intermediate & Resistant \\
Gentamycin & + & - & - \\
Chloramphenicol & + & - & - \\
Cephalexin & + & - & - \\
Kanamycin & + & - & - \\
Cephradine & - & + & - \\
Streptomycin & - & + & + \\
Tetracycline & - & - & + \\
Amoxicillin & - & - & + \\
Nalidixic acid & - & - & + \\
\hline + = yes; - = No & - & - &
\end{tabular}

\section{Discussion}

In this study, the overall prevalence of $E$. coli in chickens was recorded as $78.86 \%$. Hossain et al. (2008) reported $60 \%$ prevalence, whereas Nazir (2004) observed $62.5 \%$ prevalence of $E$. coli in chickens. In another study, 66.67\% prevalence of E. coli was noticed in duck (Avishek, 2010).

Antibiotic resistance of $E$. coli isolates of chickens recorded in this experiment might be due to indiscriminate use of antibiotics (Bonnet et al., 2009). These drug resistant $E$. coli can be spread in the environment where humans and 
animals acquire infections resulting difficulties in treating these cases (Alexander et al., 2009). The prevalence of antibiotic resistant $E$. coli observed in this study appeared to be similar to several other studies (Khan et al., 2005; Nazir et al., 2005). Chicken intestine can serve as a reservoir for E. coli strains capable of causing extra-intestinal infection in avian and mammalian hosts (Ewers et al., 2009). Clinically, healthy chickens probably have zoonotic potential since transmission between birds and humans via physical contact, contaminated dust and egg is possible. Feces tends to be leaked from the carcass when the evisceration stage itself gives an opportunity for the interior carcass to receive intestinal bacteria (Gormley et al., 2011). Contaminated meat and other foods may play a role in the local spread of E. coli strains (Vincent et al., 2010). The use of antimicrobial agents in food animal production may cause emergence of antimicrobial drug-resistant strain of E. coli. Antimicrobial drug-resistant E. coli was reported in humans due to the consumption of contaminated chicken (Manges et al., 2007).

\section{Conclusion}

Data in this study suggested that chickens from farms and live bird markets may act as major reservoir of $E$. coli in Mymensingh region of Bangladesh. Harmful isolates of $E$. coli might cause food-borne illness and may spread resistance strain in humans following consumption of contaminated poultry and poultry by-products.

\section{Acknowledgement}

This research work was funded by the Bangladesh Agricultural University Research System (BAURES) grants (Project No. 2010/32/BAU).

\section{References}

Alexander TW, T Reuter, R Sharma, LJ Yanke, E Topp and TA McAllister, 2009. Longitudinal characterization of resistant Escherichia coli in fecal deposits from cattle fed subtherapeutic levels of antimicrobials. Appl Environ Microbiol, 75: 7125-7134.

Avishek S, 2010. Characterization of Escherichia coli isolates from duck of Bangladesh and Nepal. MS Thesis. Department of Microbiology and Hygiene, BAU, Mymensingh-2202.

Barnes HJ and WB Gross, 1997. Colibacillosis. In: Diseases of Poultry. (Calnek BW, Barnes HJ, Beard CW, McDougald LR and Saif YM, eds). 10th Ed, Iowa State University Press; Ames, IA, USA, pp: 131-141.

Bauer AW, WM Kirby, JC Sherris and M Turck, 1966. Antibiotic susceptibility testing by a standardized single disc method. Am J Clin Pathol, 45: 493-496.

Bélanger L, A Garenaux, J Harel, M Boulianne, E Nadeau and CM Dozois, 2011. Escherichia coli from animal re-servoirs as a potential source of human extraintestinal pathogenic E. coli, FEMS Immunol Med Microbiol, 62: 1-10.

Bonnet C, F Diarrassouba, R Brousseau, L Masson, E Topp and S Moussa, 2009. Pathotype and antibiotic resistance gene distributions of Escherichia coli isolates from broiler chickens raised on antimicrobial-supplemented diets. Appl Environ Microbiol,
75: 6955-6962.

Cheesbrough M, 1985. Medical Laboratory Manual for Tropical Countries. Vol 2: Microbiology. Tropical Health Technology/ Butter-worth and Co. Ltd. Cambridgeshire/Kent.

Cowan ST, 1985. Cowan and Steel's manual for identification of medical bacteria. $2^{\text {nd }}$ Ed, Cambridge University Press, Cambridge, London, pp: 138-139.

Dho-Moulin M and JM Fairbrother, 1999. Avian patho-genic Escherichia coli (APEC). Vet Res, 30: 299-316.

Ewers C, EM Antao, I Diehl, HC Philipp and LH Wieler, 2009. Intestine and environment of chicken as reservoir for extra intestinal pathogenic $E$. coli strain with zoonotic potential. Appl Environ Microbiol, 75: 184-192.

Geser N, R Stephan and H Hächler, 2012. Occurrence and characteristics of extended-spectrum $\beta$-lactamase (ESBL) producing Enterobacteriaceae in food producing animals, minced meat and raw milk. BMC Vet Res, 8: 21.

Gormley FJ, CL Little, N Rawal, IA Gillespie, S Lebaigue and GK Adak 2011. A 17-year review of foodborne outbreaks: describing the continuing decline in England and Wales (1992-2008). Epidemiol Infect, 139: 688-699.

Hossain MT, MP Siddique, FMA Hossain, MA Zinnah, MM Hossain, MK Alam, MT Rahman and KA Choudhury, 2008. Isolation, identification, toxin profile and antibiogram of $E$. coli isolated from broilers and layers in Mymensingh district of Bangladesh. Bangl J Vet Med, 6: 1-5.

Khan MFR, MB Rahman, MSR Khan, KHMNH Nazir and M Rahman, 2005. Antibiogram and plasmid profile analysis of isolated poultry Salmonella of Bangladesh. Pak J Bio Sci, 8: 1614-1619.

Manges AR, SP Smith, BJ Lau, CJ Nuval, JN Eisenberg and PS Dietrich, 2007. Retail meat consumption and the acquisition of antimicrobial resistant Escherichia coli causing urinary tract infections: a case-control study. Foodborne Pathog Dis, 4: 419431.

Marshall B, D Petrowski and SB Levy, 1990. Inter- and Intra species spread of Escherichia coli in a farm environment in the absence of antibiotic usage. Proc Natl Acad Sci USA, 87: 6609-6613.

Mead PS, L Slutsker, V Dietz, LF McCaig, JS Bresee, C Shapiro, PM Griffin and RV Tauxe. 1999. Food-related illness and death in the United States. Emer Infect Dis, 5: 607-625.

Nazir KHMNH, 2004. Molecular base of diversified E. coli potentiating antibiotic resistant pattern and compromising epidemiology. MS Thesis. Department of Microbiology and Hygiene, BAU, Mymensingh-2202.

Nazir KHMNH, MB Rahman, KM Nasiruddin, F Akhtar, MFR Khan and MS Islam, 2005. Antibiotic sensitivity of Escherichia coli isolated from water and its relation with plasmid profile analysis. Pak J Bio Sci, 8: 1610-1613.

NCCLS. 2003. Performance standard for antimicrobial testing. $13^{\text {th }}$ informational supplement (Disk diffusion supplement tables). NCCLS document M100-S13 (M2), supplement to NCCLS document M2-A8 (disk diffusion).

Otaki Y, 1995. Poultry disease control programme in Japan. In: Asian Livestock (FAO), 20: 65-67.

Pupo GM, DK Karaolis, R Lan and PR Reeves, 1997. Evolutionary relationships among pathogenic and nonpathogenic Escherichia coli strains inferred from multilo-cus enzyme electrophoresis and mdh sequence studies. Infect Immun, 65: 2685-2692.

Vincent C, P Boerlin, D Daignault, CM Dozois, L Dutil, C Galanakis, RJ Reid-Smith, PP Tellier, PA Tellis, K Ziebell and AR Manges, 2010. Food reservoir for Escherichia coli causing urinary tract infections. Emerg Infect Dis, 16: 88-95. 